
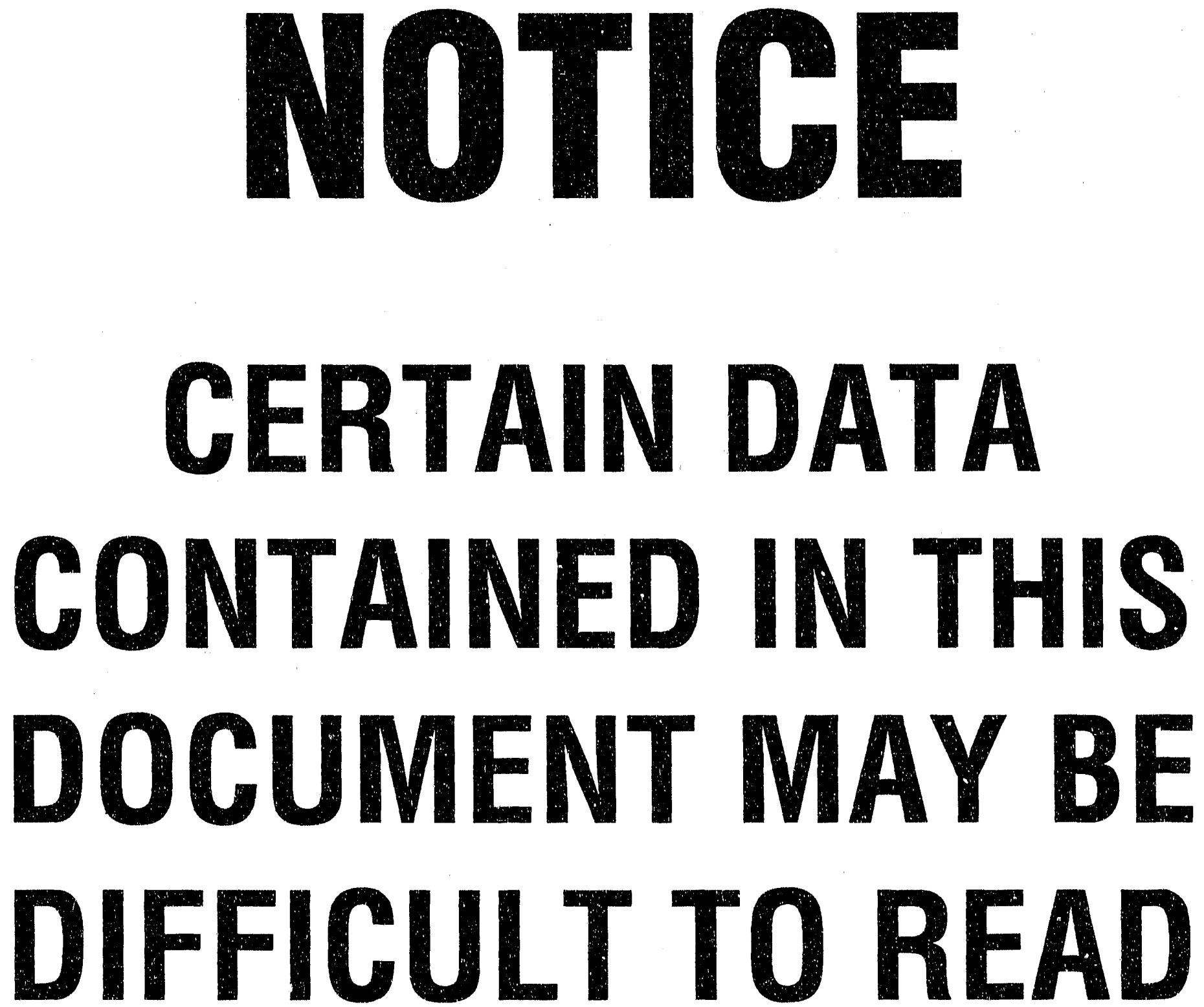

IN MICROFICHE

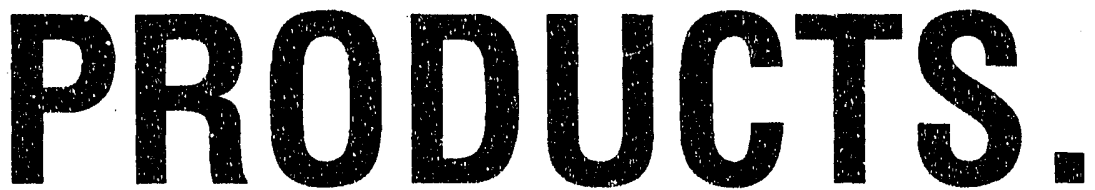




\section{TTILE: JASA: A Prototype Water-Cerenkov} Air-Shower Detector

Author(s): D. Berley, C. Dion, J.A. Goodman, T.J. Haines, P.W. Kwok, M.J. Stark, R.C. Svoboda, H. Ferguson, C. M. Hoffman, E. P. Horch, R. W. Ellsworth

SUBMrTten TO: International Conference on High Energy Gamma Ray Astronomy Ann Arbor, Michigan, Oct. 2 - 5, 1990

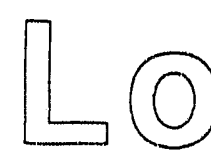

S) (A) 亩 กิก<smiles>[18OH]</smiles>
S Los Alamos National Laboratory Los Alamos, New Mexico 87545

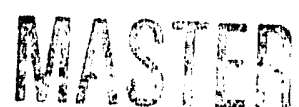

13

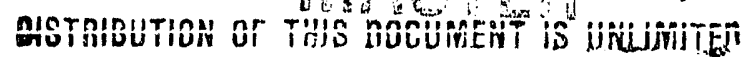




\title{
JASA: A PROTOTYPE WATER-ČERENKOV AIR-SHOWER DETECTOR
}

\author{
D. Berley, C. Dion, J.A. Goodman, T.J. Haines, P.W. Kwok, M.J. Stark \\ The University of Maryland, College Park \\ R.C. Svoboda \\ Louisiana State University, Baton Rouge \\ H. Ferguson, C.M. Hoffman, E. Horch \\ Los Alamos National Laboratory, Los Alamos, NM \\ R.W. Ellsworth \\ George Mcson University, Fairfax, VA \\ R.S. Delay, X-Q. Lu, G.B. Yodh \\ The University of California, Irvine
}

\begin{abstract}
A small pilot experiment to examine the use of the water-Cerenkov technique for air shower detection was installed near the center of the CYGNUS air shower array. Preliminary results showing general agreement with simulations are presented. Thus, the technique promises to offer significant advances for VHE-UHE $\gamma$-ray astronomy.

Sparse arrays of scintillator detectors have been used for several decades to study the properties of extensive air showers. Most recently, these arrays have been used in the search for point sources of UHE gamma-rays.[1] In light of recent results, there is clearly a continuing need to improve the sensitivity of these experiments. An obvious way to lower an array's threshold, shown below to improve its sensitivity, is to simply pack scintillation detectors close together; this hes been examined[2] and shown not to be a viable alternative because the resulting angular resolution is not very good and the cost of building the array is high. During the last decade, the water-Cerenkov technique has proven to be a cost effective method for large, fully sensitive particle detectors, best illustrated by the successful IMB and Kamiokande underground experiments. Large surface water-Čerenkov detectors offer a new technique for air shower detection as well.

The sensitivity of an experiment measuring cosmic $\gamma$-ray's depends on several factors: the total area, the energy threshold, the angular resolution, and the ability to reject background. The number of events from a source during an observing time $T$ is

$$
\text { No } \propto F, A_{f}^{e f f} T
$$

where $F_{,}$, the source flux, does not depend on the experimental situation and $A_{f}^{\text {eff }}$ is the effective area of the array (the area times the integral of the flux times the efficiency integrated over energy and zenith angles) above the threshold energy. The number of background events in a bin of radius $\delta \theta$, the angular resolution, is

$$
\mathrm{N}_{c r} \propto \mathrm{F}_{c r} \mathrm{~A}_{c t}^{e f t} \mathrm{~T}\left(\delta \hat{e}^{\prime}\right)^{2} / \mathcal{F}
$$

where $\mathcal{F}$ is a possible background rejection factor due to, for example, a cut only allowing muon poor showers. Typically $\mathrm{A}_{s}^{e f f} \approx \mathrm{A}_{c r}^{e f f}$ since they only have a slight dependence on the different spectra and primary particle type; let $\mathcal{A}$ represent the ratio $\mathrm{A}_{j}^{\text {eff }} / \mathrm{A}_{c r}^{e f f}$, which can depend on the experimental situation but has a typical value of 1 to 3 . In addition, the trigger rate due to cosmic rays $\left(R_{c r}\right)$ is proportional to $A_{c f}^{e f f}$. Thus,
\end{abstract}




$$
\mathrm{N}, \propto \mathrm{R}_{\text {er }} \mathcal{A} \mathrm{T}
$$

and

$$
\mathrm{N}_{c r} \propto \mathrm{R}_{c r} \mathrm{~T}(\delta \theta)^{2} / \mathcal{F}
$$

where the factors that have been left out do not depend on the experimental situation. The statistical significance of ar given source strength observed for a given period of time is a "figure-of-merit." This is given by

$$
\frac{s}{\sqrt{N}} \propto \sqrt{R_{\text {cr }} \mathcal{F} \mathcal{A}} / \delta \theta
$$

with the ratio on the right representing the "quality" of an experiment.

The cosmic ray rate depends roughly linearly on the area and the -1.7 power of the energy threshold; thus, the quality depends only on the square root of the area but nearly linearly on both the threshold and angular resolution. It then follows that an experiment may be improved most rapidly by either lowering the threshold or improving the angular resolution, or both. It is in providing the potential improvement of both of these aspects that the water-Čerenkov technique holds most promise for the next generation detector.

The idea of a large area water-Čerenkov detector deployed for gamma-ray (and neutrino) astronomy was first developed for the $30,000 \mathrm{~m}^{2}$ GRANDE experiment[3]: simulations of the detector response to air showers shows the potential for reduced threshold and improved resolution with the added advantage of muon and hadron detection over its entire surface. The reason for this is that a water-Cerenkov detector is sensitive over its entire surface to all components of the air shower: the electronic, photonic, muonic, and hadronic components will all be observed. Thus, the detector is potentially sensitive to lower energy showers and is capable of reconstructing them with better angular resolution. More recently a high-altitude detector, called MILAGRO,[4] has also been proposed for VHE-UHE gamma-ray astronomy; the high altitude location of this experiment offers a further reduction in energy threshold.

Thus, there is a clear need to perform a pilot water-Čerenkov experiment to verify the simulations of the detector response. The obvious advantage of performing the pilot experiment in coincidence with an existing air shower array is that the air shower array can determine many of the characteristics of each shower that the water detector observes: the direction, shower size, and core location can all be determined by the air shower array with sufficient accuracy.

Such a pilot experiment, named JASA, has begun near the center of the CYGNUS air shower array in Los Alamos, NM at an altitude of $2,100 \mathrm{~m}$. Some of the mosi relevant features of the arroy will be given here with more details of the CYGNUS experiment found elsewhere.[5] The pilot experiment is deployed in the center of the CYG-I array, shown in Figure 1; this part of the array, which covers about $20,000 \mathrm{~m}^{2}$ of the $80,000 \mathrm{~m}^{2}$ total ares of the array, is the most densely covered with 108 detectors of typical separation $15 \mathrm{~m}$. Each detector is about $1 \mathrm{~m}^{2}$ of scintillator viewed by a cingle phototube. The angular resolution of the CYGNUS array is about $0.75^{\circ}$, the energy threshold is about $50 \mathrm{TeV}$, and the trigger rate is currently about 6 events per second.

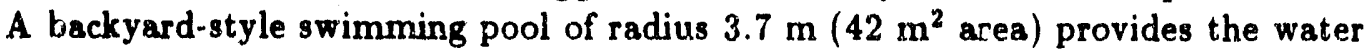
enclosure for the pilot experiment; a light-tight black PVC cover and black plastic lining assure minimal light reflection from the inner surfaces of the pool. The total depth of 


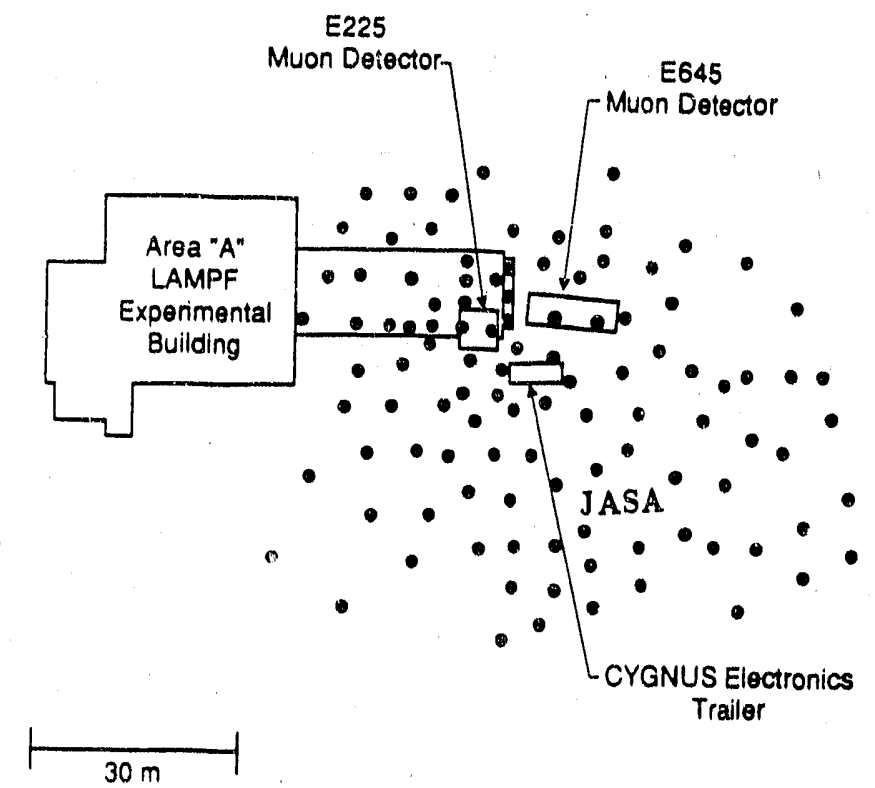

Figure 1: Deployment of counters in CYG-I; esch point represents a counter. Also shown is the location of the water-Cerenkov detector.

the pcol, about $2.3 \mathrm{~m}$, provides $2 \mathrm{~m}$ of water above the faces of the photomultiplier tubes (PMTs); the arrangement of the pool is shown schematically in Figure 2. There are currently 10 8" Hamamatsu PMTs, borrowed from the IMB collaboration, located near the center of the pool; 2 additional 10" Burle PMTs, with much better timing characteristics (jitter $<2.5$ ns FWHM), will be placed in the pool to improve the timing measurements.

The pool was run in coincidence with the CYG-I air shower array for about 8 hours, long enough to collect about 120,000 CYG-I events; preliminary results from these data will be given here with more refined results still to be completed.

A simple illustration of the power of a water-Čerenkov detector is seen by noting that each PMT in the pool detects ( $\geq 1$ photoelectron) at least $95 \%$ of all oi the showers that triggered CYG-I, including showers at large zenith angles and large core distances; taken together, the PMTs in the pool detect at least $99.7 \%$ of all of the showers. The singles rates of the PMTs in the pool vary between about $15 \mathrm{kHz}$ and $30 \mathrm{kHz}$, in general agreement with the expected rate of about $20 \mathrm{kHz}$ at the altitude of Los Alamos. The coincidence rate of PMTs in the pool $(\sim 10 \mathrm{kHz})$ is very high because it is possible for a single muon to trigger all 10 PMTs; this same problem will be faced by the large area detectors and must be dealt with in some detail to define actual triggering schemes.

The core position, direction, and shower size of each of the events was determined from the CYG-I data; typical uncertainties are $<5 \mathrm{~m}$ in core position and about $50 \%$ in shower size. The radial dependence of the Čerenkov light observed in the pool is determined by examining showers with approximately fixed shower size $\left(10,000<\mathrm{N}_{e}<a n, 000\right.$, corresponding to $\sim 50 \mathrm{TeV}$ showers) from near the zenith. The average number of photoelectrons recorded in the pool, as a function of core distance, is shown in Figure $3 ;$ the 


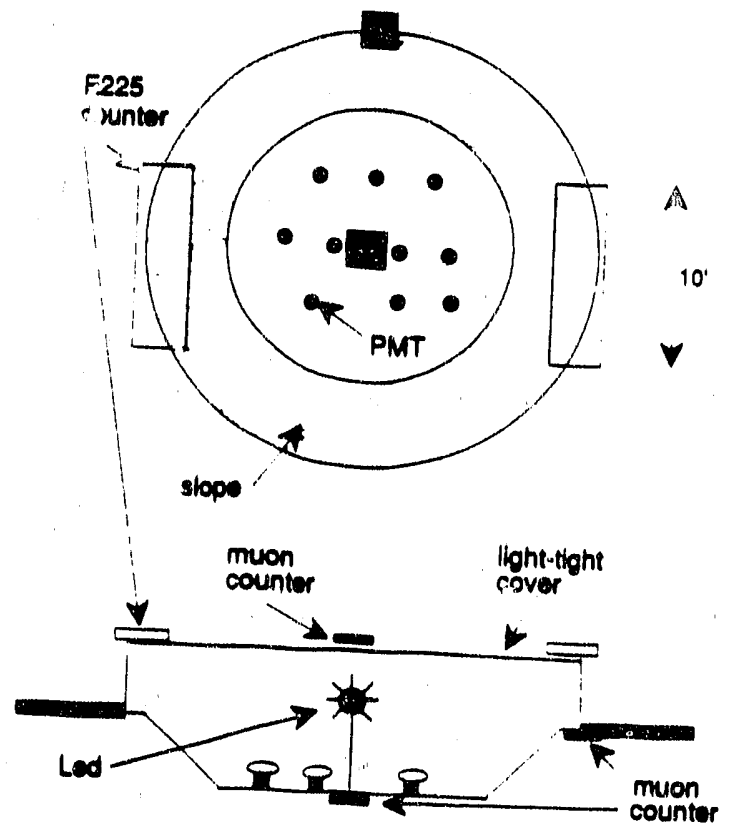

Figure 2: Schematic showing the arrangement of the PM'Ts in the pool. Also shown are auxiliary scintillation detectors for local particle measurement, an LED and muon detectors for calibration.

absolute amount of light, as determined from a calibration of the PMT pulse heights, is now known to only about $25 \%$.

A relatively crude simulation of the expected response of the pool, using some of the same Monte Carlo programs that were used to simulate both the GRANDE and MILAGRO detectors, was made to check against the data recorded in the pool. Several very important effects, such as core position and shower size errors, were ignored in this preliminary study. This can cause systematic errors, especially in the presence of the steeply falling cosmic ray spectrum. Nevertheless, the general agreement between the simulation and the data, also shown in Figure 3, suggests that the low energy threshold of a water-Čerenkov detector predicted by the simulation is probably correct.

A large improvement in angular resolution over conventional air shower arrays is also predicted by the simulations, again due to the fact that the air shower is sampled more finely by a Cerenkov detector. The angular resolution depends critically on the timing of the air shower front, the timing ability of the PMTs, as well as the particular geometry of the Cerenkov light pattern which depends, in turn, on the energies and directions of the shower particles. The timing characteristics of the PMTs used in the pool are somewhat poorer than the current generation of large PMTs. The width of the timing signal, for the same sample of events as above, is shown in Figure 4 ; the average rms width for all showers is about $4 \mathrm{~ns}$, again in reasonatle agreement with the predictions of the simulation. At a core distance of about $100 \mathrm{~m}$, the average timing width is about $6 \mathrm{~ns}$.

The results are encouraging enough that several additional pools will be deployed around the edges of the CYG-I array early in 1991. The goal of this effort is to improve 


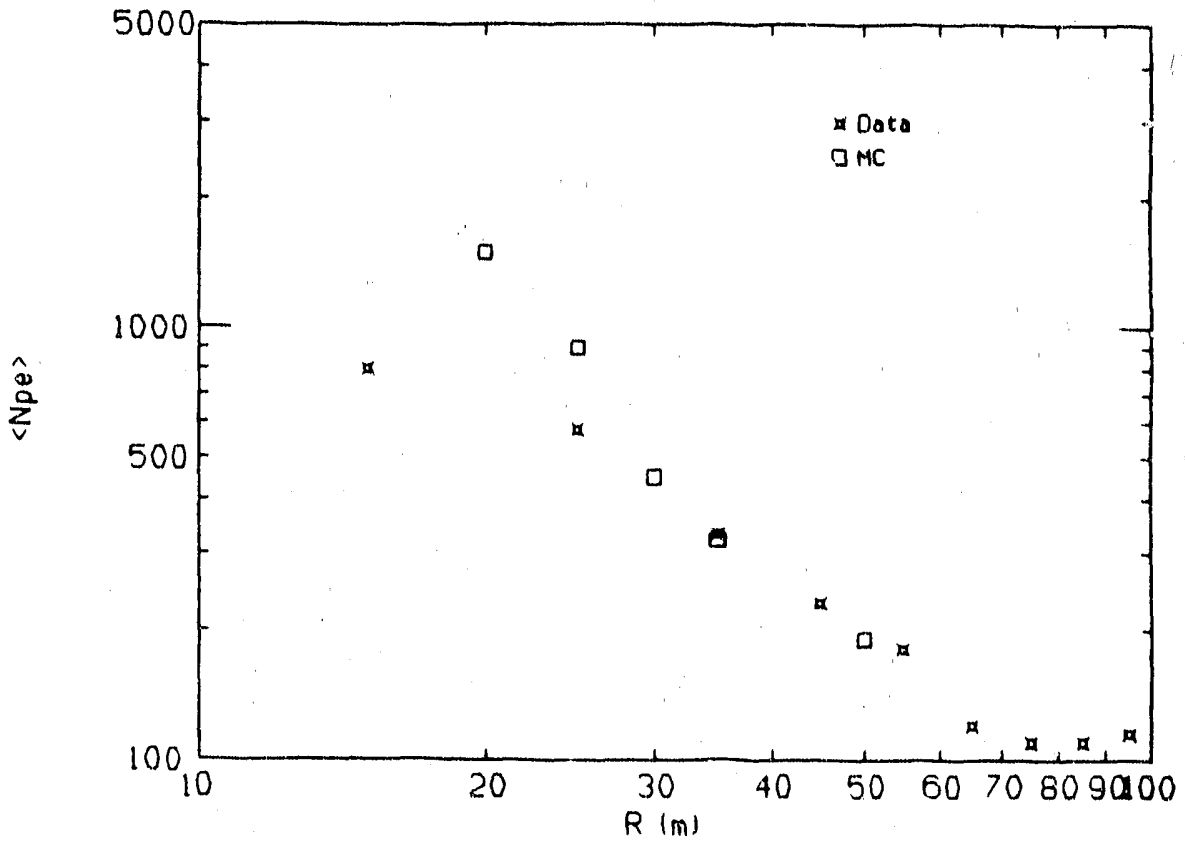

Figure 3: The number of pe's observed in the pool ss a function of core position. Also shown are the results of a relatively crude simulation.

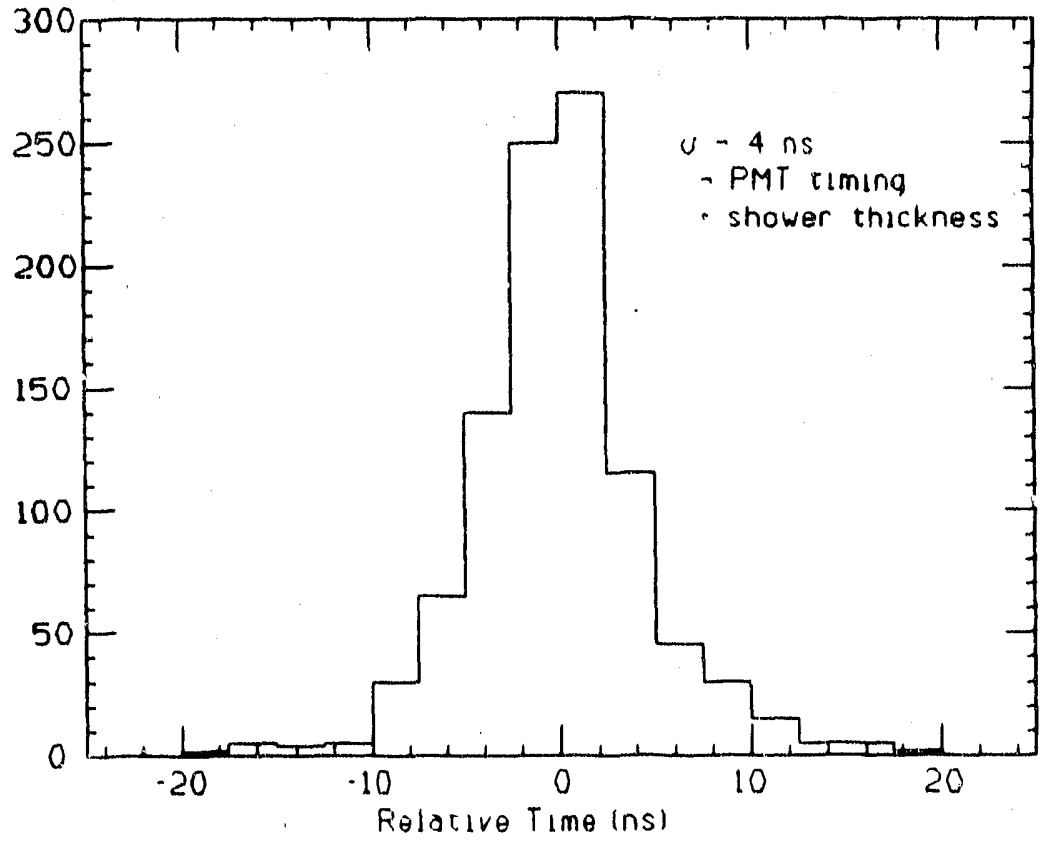

Figure 4: The relative time of each PMT averaged over many showers. 
the angular resolution of the CYGNUS experiment by using the timing information recorded in the pools. A semi-emperical formula that relates the timing of a detector element in an air shower array (including shower and detector timing together), $\sigma_{t}$, the lattice spacing of the elements, $\mathcal{L}$, and the number of elements used in reconstruction, $\mathrm{N}$, that has been verified with CYGNUS data is

$$
\delta \theta(\mathrm{rad}) \simeq \frac{\sigma_{1}(\mathrm{ne})}{N \mathcal{L}(m)} \text {. }
$$

Applying this formula to the case of four pools separated by $100 \mathrm{~m}$, each pool having nine PMTs so the time of the air shower recorded in each pool is $\sigma_{t} \simeq 2 \mathrm{~ns}(6 \mathrm{~ns} / \sqrt{9})$, yields an angular resolution of about $0.3^{\circ}$.

Though more detailed studies must be done, the performance of the pilot experiment is in rather good agreement with the expectations based on Monte Carlo simulations of the air shower and the response of the detector to the air showers. This lends important experimental support to the idea that large area air shower arrays based on the water-Cerenkov technique hold great promise in advancing the field of VHE-UHE $\gamma$-ray astronomy into the future.

We would like to thank the IMB collaboration for lending us the PMTs used in this experiment, and our many hard working colleagues in the CYGNUS collaboration. We would also like to thank Robin Barrone and Matt of Matt's Pools. This work was supported in part by the National Science Foundation and the U.S. Department of Energy.

\section{References}

[1] For a recent review please see D.J. Fegan, Proc. of the XXI Int'l. Cosmic Ray Conf., R.J. Protheroe, ed., (U. Adelaide) 11, 23(1990).

[2] T.J. Haines et al., Proc. of the Wurkshop on the Physics an Experimental Techniques of High-Energy Neutrino and VHE and UHE Gamma-Ray Particle Astrophysics, G. Yodh, D. Wold, and W. Kropp, eds., Nucl. Phys. B-Proc. Supp. 14A, 244(1989).

[3] T.J. Haines et al., these proceedings.

[4] J.A. Goodman et al., these proceedings.

[5] R.W. Ellsworth et al, these proceedings.

\section{DISCLAIMER}

This report was prepared as an account of work sponsored by an agency of the United States Government. Neither the United States Government nor any agency thereof, nor any of their employees, makes any warranty, express or implied, or assumes any legal liability or responsibility for the accuracy, completeness, or usefulness of any information, apparatus, product, or process disclosed, or represents that its use would nnt infringe privately owned rights. Referprocess disclosed, or represente that its use would $n$ ess, or service by trade name, trademark, manufacturer, or otherwise does not necessarily constitute or imply its endorsement, recornmendation, or favoring by the United States Government or any agency thereof. The views and opinions of authors expressed herein do not necessarily state or reflect those of the United States Government or any agency theieof. 

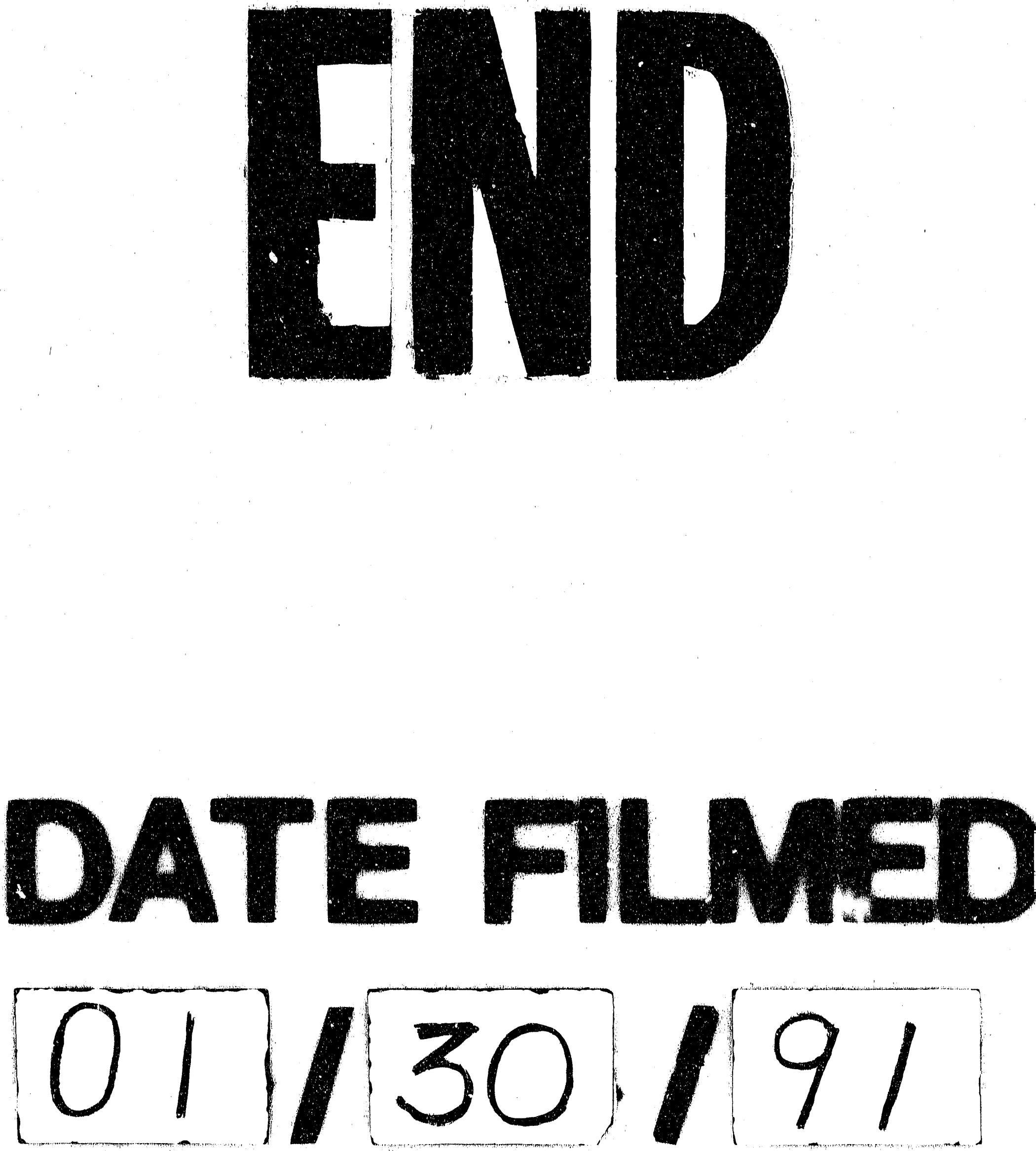
I 